\title{
Critical Sized Mandibular Defect Regeneration in Preclinical In Vivo Models
}

\author{
G. I. Brierly ${ }^{1} \cdot$ S. Tredinnick ${ }^{2} \cdot$ A. Lynham $^{3}$ - M. A. Woodruff $^{3}$
}

Published online: 18 April 2016

(C) Springer International Publishing AG 2016

\begin{abstract}
Restoration of critical sized defects of the mandible still poses a major problem in the field of Oral and Maxillofacial Surgery. The current gold standard of treatment relies on the use of autologous grafts and therefore, has associated disadvantages such as donor site morbidity and insufficient bone for prosthetic rehabilitation. Recent advances in the fields of bone tissue engineering and distraction osteogenesis provide an alternative treatment modality to restore the aforementioned critical sized defects. These alternative modalities offer patients and clinicians multiple advantages such as decreased morbidity and increased volume of bone for prosthetic restoration. The aim of this review was to evaluate the bone regeneration capacity of bone tissue engineering and distraction osteogenesis in preclinical in vivo animal models against the current gold standard.
\end{abstract}

Keywords Mandible $\cdot$ Regeneration $\cdot$ Animal model · Scaffold $\cdot$ Critical size defect $\cdot$ Growth factor

This article is part of the Topical Collection on Preclinical Animal Models in Regenerative Medicine

M. A. Woodruff

mia.woodruff@qut.edu.au

1 School of Medicine, University if Queensland, Brisbane, QLD, Australia

2 Department of Orthopaedic Surgery, University of Otago, Christchurch, New Zealand

3 Institute of Health and Biomedical Innovation, Queensland University of Technology, 60 Musk Ave, Kelvin Grove, QLD 4049, Australia

\section{Introduction}

The restoration of critical-size bone defects is a major problem for Oral and Maxillofacial Surgeons. A critical-size defect will not heal spontaneously [1] or regenerate more than $10 \%$ of the lost bone during the patients lifetime [2]. The ideal mandibular reconstruction will restore form, function, sensation, mastication and aesthetics. Modalities to treat critical-sized mandibular defects include micro-vascular free flaps, autogenous bone grafts, bridging plates and transport distraction osteogenesis [3•]. The best treatment modality should be selected based on the disease process, the anatomy involved, and the required properties of the reconstruction [3•].

Current techniques approved for use on patients fall short of the ideal treatment $[4,5]$. Immediate vascularized bone and soft tissue transfer is the current benchmark for the reconstruction of critical-size defects of the mandible following cancer resection. Although this technique has proven successful, it has multiple disadvantages including increased patient morbidity, a limited volume of transported bone and the need for a second operative site [4]. Due to the disadvantages of autogenous tissue transfer [4], focus has shifted towards the development of other treatment modalities. These new treatments may achieve ideal mandibular reconstruction with lower patient morbidity compared to the current benchmark.

Transport-disc-distraction osteogenesis (TDDO) is an alternative treatment for the reconstruction of segmental mandibular defects [6]. TDDO involves the gradual movement of a mobilized bone disc to restore continuity of the mandible over several months, without the need for a second operative site [6]. However, as aesthetics are compromised due to pin tracks, this technique does not satisfy all the criteria of ideal mandibular reconstruction [6]. Additionally, TDDO relies on patient compliance and there is uncertainty regarding efficacy in areas of previous or pending irradiation [6]. 
Another emerging treatment is tissue engineering (TE). TE is the application of scientific principles to the design, fabrication, modification and growth of living tissues using biomaterials, cells and growth factors [7]. The aim of bone tissue engineering is to regenerate lost bone via the use of growth factors and/or cells. This approach could potentially obviate the need for a second operative site and provide bone volume and contour necessary for cosmesis and the restoration of occlusion.

In bone TE, an appropriate carrier can deliver osteogenic growth factors and autogenous bone precursor cells to a defect site. Typically, these carriers are three dimensional scaffolds that facilitate cell attachment and proliferation, and can themselves be used as a vehicle for growth factor delivery [8]. The addition of cells and growth factors into scaffolds can result in a repair site that is both osteoinductive and osteoconductive [9]. Some effective combinations of osteogenic growth factors and autogenous bone precursor cells in scaffolds have been investigated to promote bone regeneration in critical sized defects $[10,11]$.

Preferably, TE materials should be biocompatible and not elicit a foreign body reaction. The scaffold should have an anatomical shape that is porous to allow for the infiltration of cells and the free movement of waste products. Critically, they should promote cell adhesion and proliferation on and into the scaffold structure [12]. Scaffolds must also be mechanically robust to withstand physiological stresses at the site of implantation.

Growth factors should stimulate bone healing at an acceptable dose concentration via the recruitment of osteogenic factors and the promotion and differentiation of osteogenic progenitor cells [11]. Moreover, growth factors must achieve an ideal time of localization at the defect site whilst maintaining a concentration sufficient to promote bone healing [11]. Growth factors may be incorporated in the scaffold material itself or delivered separately.

The ideal method of de novo bone synthesis using TDDO or a TE scaffold must be determined with pre-clinical in vivo testing. This testing must be conducted in an appropriate animal model with rigorous post euthanasia analysis [13]. Only after extensive testing and appropriate long-term review can clinicians and researchers be confident that the new modality is both effective, safe and meets the criteria for ideal reconstruction.

Animal models with continuity and non-continuity defects are used to investigate mandibular reconstruction. Non-continuity defects do not require fixation and are typically circular or rectangular resections, whilst continuity defects are typically segmental resections and result in loss of mandibular unity. Thus, fixation is required for continuity defects and the resulting model is more complex yet, more accurately mirrors the clinical condition.
This report reviews the relevant literature concerning regeneration or reconstruction of critical sized mandibular defects in animal models. Furthermore, this review aims to highlight the current modalities that show promise and the future direction of mandibular reconstruction in animal models.

\section{Bone Transport Disc Distraction Osteogenesis}

Transport disc distraction osteogenesis (TDDO) is a specialized technique used to grow bone across a defect. Using TDDO, a bone disc is prepared adjacent to the defect and moved slowly and continuously until docking after the entire defect has been spanned [6]. Once docking has been achieved the regenerated bone undergoes remodeling and calcification. TDDO provides an alternative treatment option for patients who have lost mandibular continuity, without the need for a second operative site [6]. Costantino, in particular paved the way for TDDO in animal models. Using dogs the group were able to demonstrate regenerated bone that was $77 \%$ the strength of the contralateral mandible and therefore, could withstand the loads associated with mastication $[14,15]$.

Further animal studies have been conducted examining the application of TDDO for regeneration of mandibular segmental defects [16]. In all the animal models, the critical sized defects were bridged with regenerated bone however, only three of the studies reported similar bone density to the native bone, possibly due to the increased docking times of the studies [17-19]). Despite regeneration of the critical sized defect, the use of TDDO was not without complication. Complications encountered included infection and fracture of the fixation plates [17], tilting of the transport bone disc, mechanical malfunction of the distraction device, regenerated bone fracture, and wound dehiscence $[20,21]$. The most concerning complications reported were problems with transport disc docking. In one study, calcific material formed between the docking site and the transport disc impeded the distraction. However, this did not result in a non-union. Other studies reported high rates of non-union at the docking sites of the transport disc due to coned shaped bone outgrowths preventing adequate adaption of bone sites on contact. This led to failure rates ranging from $50 \%$ [21] to $100 \%$ [20] of animals. Thus, despite the benefits afforded with the use of TDDO, there still remains doubt regarding the strength of the regenerated bone, and the predictability of bone disc docking in animal models.

\section{Tissue Engineered Constructs}

\section{Non-continuity Defects}

Non-continuity defects of the mandible maintain the unity of the mandible, do not require fixation and are typically circular or rectangular three walled defects. These mandibular defects 
are often used in small animal models and provide necessary information regarding biocompatibility, toxicity and the bone regenerative capacity of novel bone tissue regenerative techniques. Typically, these defects do not adequately mimic the clinical scenario of tumor or cancer resection and as such, may be better models for periodontal fenestration defects.

Novel methods targeted at treatment of critical sized defects often apply bone tissue engineering. The principles of bone tissue engineering revolve around the theme of biomimicry whereby, the regenerative capacity of the body is harnessed through the use of stimulatory growth factors or autogenous bone precursor cells with an appropriate scaffold to facilitate the restoration of bone at the site of deficiency.

A number of scaffold and cell or growth factor combinations have been trailed in animal models in an attempt to regenerate these non-continuity critical sized defects. The techniques range from the use of high compressive strength bioceramics such as hydroxyapatite seeded with bone marrow stem cells [22], calcium phosphates [23], and hydroxyapatite and collagen seeded with adipose stem cells [24], to lower compressive strength natural polymers such as platelet rich fibrin glue with mesenchymal stem cells [25], adipose derived stromal cells with fibrin glue pellets [26•] and finally, synthetic polymers like nanohydroxapatite polyamide with or without bone marrow stromal cells [27].

As evidenced by the heterogenous nature of the tissue engineering approaches, non-continuity critical sized defects are much more forgiving, allowing investigation of high and low strength materials. In all of the above studies, scaffolds combined with a growth factor or naive cells produced adequate bone regeneration with the maximum defect restoration reaching $90 \%$ [26•]. Despite the high percentage, the central region of ossification in this study lacked contact with peripheral bone. Furthermore, the studies investigating noncontinuity critical sized defects were conducted using a variety of small and large animal models, employing differing defect sizes in various anatomical sites. To compound the problem of comparison between bone tissue regenerative techniques, sacrifice times between the studies varied greatly fluctuating from 2 weeks [27] to 32 weeks [25]. Therefore, direct contrast between bone tissue regenerative techniques in animal models remains fraught with difficulties due to the inherent differences between studies.

\section{Continuity Defects}

Continuity defects are typically due to segmental resections and result in a loss of mandibular unity. These types of defects are challenging to treat as the defect will not undergo spontaneous healing thus, fixation is required to maintain the anatomic form of the mandible after resection with or without autogenous bone grafts, or vascularized osteocutaneous grafts. Due to the surgical difficulty, large animals are typically used to examine regeneration of these defects, although rabbits have been used without plate fixation [28•, 29•]. Outcomes of the interventions were most commonly assessed using histological, histomorphometric, and computed tomography techniques. Additionally, biomechanical testing was used in conjunction with the aforementioned techniques.

\section{Bone Morphogenetic Protein-2 Studies}

Recombinant human bone morphogenic protein-2 (rhBMP-2) is a member of the TGF- $\beta$ superfamily and has a proven ability to promote bone growth [30]. The combination of rhBMP-2 with an appropriate carrier is intended to localize the growth factor within the defect site and enhance tissue formation. Multiple authors have employed the use of rhBMP-2 in combination with an appropriate carrier in order to regenerate defects within the mandible. The use of rhBMP-2 was studied in combination with resorbable collage [31, 32], collagen sponge with hydroxyapatite and $\beta$-Tricalcium Phosphate crystals [33•] and a poly D,L-lactic-co-glycolic-acid coated gelatin sponge [34]. The use of poly D,L-lactic-co-glycolic-acid coated gelatin sponge reported the best result, yielding adequate bone width and height within the defect after 30 weeks, as well as lamellar bone remodeling within the $30 \mathrm{~mm}$ defect. It should be noted however, that $9 \mathrm{mg}$ of rhBMP-2 was used. The use of rhBMP-2 with a collagen sponge produced $56.3 \%$ defect filling, although this was only 4 weeks after implantation [34].

\section{Autologous Bone Marrow Derived Stromal Cells Studies}

Bone marrow derived stromal cells can be grouped under the term mesenchymal stem cells (MSCs). These multipotent stromal cells have the ability to differentiate into a variety of cells types that produce many critical tissues. The use of MSCs offers another potential treatment modality for the repair of critical size defects [35]. Autologous bone marrow derived stromal cells proved to be a popular choice among authors attempting regeneration of critical sized continuity defects in the mandible. MSCs were seeded onto a variety of scaffolds including $\beta$-Tricalcium Phosphate [29•, 36, 37, 38•, 39], pyrolized bovine bone scaffolds [40] and coral [38•]. Of the $\beta$ Tricalcium Phosphate scaffolds, the study by Yuan et al. produced the most encouraging results, achieving bone union at 26 weeks in a $30-\mathrm{mm}$ defect with comparable bone mineral density to the contralateral mandible. These results were overshadowed by the coral scaffolds however, which achieved bone bridging in the mandible at 26 weeks, and at 32 weeks had higher bone mineral density and comparable bending load strength compared to the contralateral unoperated mandible. However, not all the studies using mesenchymal stem cells produced favorable results. Alfotawei et al. [29•] did not find any significant improvement in bone reconstruction following the administration of bone marrow stem cells, and Schliephake 
et al. reported bone formation mainly in the marginal sections of the defect with $25 \%$ of the animal subjects having fractured their fixation plates [40] .

\section{Bone Morphogenetic Protein-7, Cancellous Bone Graft Studies}

Bone morphogenetic protein-7 (BMP-7), like bone morphogenetic protein-2 (BMP-2), is a member of the transforming growth factor-beta (TGF- $\beta$ ) superfamily [10]. Both BMP-2 and BMP-7 are known for their osteoinductive and chrondroinductive properties and the former has been FDA approved for use in procedures such as maxillary sinus augmentation [30]. The authors who conducted regeneration and reconstruction on the mandible, employed the use of rhBMP-7 and cancellous bone grafts as their osteogenic stimulus [28•, 41]. A number of different carriers were used in conjunction with rhBMP-7 $(3.5 \mathrm{mg})$, with the most popular being type-1 collagen scaffolds [42-44]. Other regenerative techniques employed include the use of an resorbable alloplastic bone tray with cancellous bone grafts from the iliac crest [41], rhBMP-7 in a bovine collagen type-1 sponge wrapped in a pedicled sterno-occipitalis muscle flap [43] and a $\beta$-TCP scaffold with rhBMP-7 (400 ng) in a bovine bone collagen type-1 carrier [28•]. The studies using rhBMP-7 were not associated with predictable defect bone bridging. Results indicated lack of homogenous bone regeneration [44] and rather calcification with soft tissue islands [43], with multiple animals lacking union between the new graft and the host bone [28 • 42-44]. The use of cancellous bone from the iliac crest produced bone bridging within the $20 \mathrm{~mm}$ segmental defect with results indicating higher mechanical strength without the use of the alloplastic bone tray [41] (Fig 1).

\section{Conclusions}

Due to recent advancements in tissue engineering, and through work carried out by Ilizarov et al. [45], there have been great steps towards the development of less morbid treatment strategies that provide better functional outcomes for patients suffering from critical size defects of the mandible. Despite these improvements in technology, vascularized bone grafts still continue to be the mainstay for treatment of critical size defects of the mandible.
Well-designed in vivo animal models continue to play an important role in helping to understand the mechanisms of mechanical intervention healing, development of translational research and tissue engineered bone formation, including the quality of the regenerate and the viability of the treatment method. The many factors which need to be taken into consideration when selecting an animal model are out of the scope of this review [13]. However, when establishing a critical sized defect model of the mandible, the etiology of the defect plays a crucial role.

The etiology of critical sized defects in the mandible can be attributed to a number of conditions ranging from malignancies such as squamous cell carcinoma, chondrosarcoma, osteosarcoma, as well as benign conditions such as fibrous dysplasia, or juvenile active ossifying fibroma [46]. Segmental mandibular defects can also be caused by conditions such as osteoradionecrosis, the use of bisphosphonates, gunshot wounds, or other trauma [47•]. Therefore, to assess the reconstructive and regenerative capacity of novel treatment techniques, research should strive to emulate the human clinical condition. Hence, use of continuity defects in animal models is vital to portray a viable clinical model.

Critical sized defect regeneration in the mandible provides important evidence regarding scaffold, cell and growth factor combinations. However, due to the anatomical locations and nature of the defects created, biomechanical testing was not carried out in all of the animal studies. Whilst $\mu \mathrm{CT}$, radiographic, histological and histomorphometric analysis can provide some information inferring the strength of the regenerate, biomechanical testing is imperative to quantifying the regenerated bone properties against controls.

Critical sized defects maintaining continuity of the mandible are usually performed in smaller animals such as rabbits or rats, which typically yield a higher statistical value and provide valuable information with regard to developing novel treatment concepts. Unfortunately, due to their differing bone structure and metabolism, it is difficult to extrapolate results to humans [48]. Consequently, small animal models play an important role in high-powered proof of principle studies that try to establish ideal scaffold/carrier and growth factor combinations. Resultant regenerative methods ultimately need to be assessed in large animal models to elucidate clinically relevant results.
Fig. 1 Creation of segmental mandibular defect in the body of the mandible. a After adjustment of the titanium plate, an osteoperiosteal segmental mandibular defect of $30 \mathrm{~mm}$ length was made at right side. $\mathbf{b}$ The defect was filled with BMSCs/ $\beta$-TCP construct [39]. Reproduced with permission from Elsevier Inc.
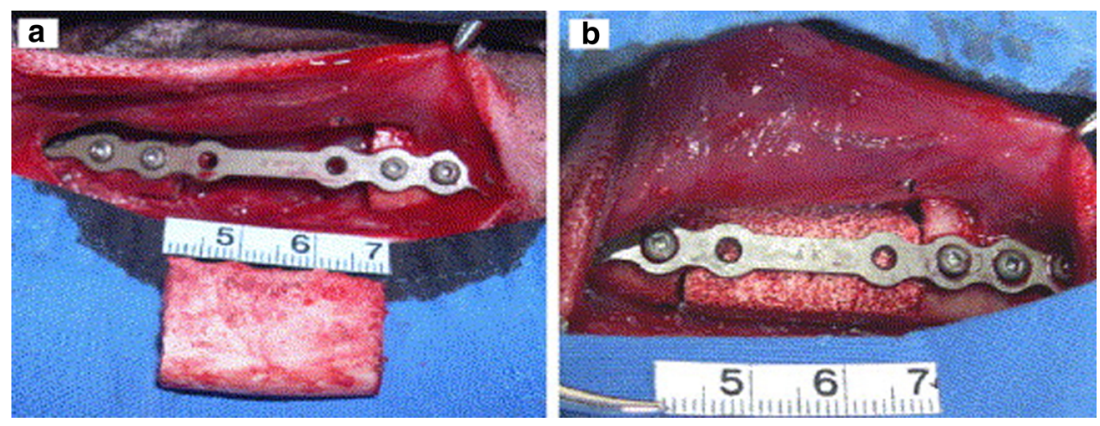
The articles which evaluated transport-disc-distraction osteogenesis used either internal or external distractors to attempt to bridge critical sized defects [17, 18, 21, 49, 50]. Authors carrying out TDDO on defects greater than $30 \mathrm{~mm}$ reported non-union rates between subjects at 50-100\% due to inadequate contact of the transport disc with the docking site $[20,21]$. These results correlate with the literature which highlight non-union of the transport disc at the docking site as a potential problem of TDDO [51, 52].

Contrary to the expected results of TDDO, two of the evaluated articles reported sound union of the transport disc with the docking site $[17,18]$. Care should be taken when interpreting these results. One article employed a defect in a small animal model that maintained the continuity of the mandible [18]. The other article, whilst using a continuity defect in a large animal model, had a small sample size of six animals due to the fracture of plates in two of the eight subjects [17]. Of those six animals, the transport disc was distracted only $15 \mathrm{~mm}$ of the $25 \mathrm{~mm}$ defect due to a $10 \mathrm{~mm}$ bone growth from the distal segment of the defect [17]. Although the regenerate yielded adequate biomechanical results, further TDDO studies should be undertaken in large animal models to validate docking site union in critical size continuity defects of the mandible. Therefore, there is currently insufficient evidence to justify the use of TDDO to repair critical sized defects in the mandible ahead of the current gold standard of vascularized bone grafts.

Of the studies which investigated restoring critical size continuity defects of the mandible, the authors primarily carried out restoration using autologous bone precursor cells or osteogenic growth factors, seeded onto osteoconductive scaffolds or sponges, compared with autogenous grafts, allografts, unseeded scaffolds, or empty defects. All results of the studies reported some form of bone bridging of the defect. Experimental groups employing the use of autogenous bone precursor cells or osteogenic growth factors, on scaffolds or sponges, produced regenerated bone with higher bone composition and better mechanical properties than scaffolds or sponges alone which is congruent with the literature [53].

These results are to be expected as the bone regeneration efficacy of BMSCs, rhBMP-2 and rhBMP-7, have been extensively documented in the literature [54]. Nevertheless, when interpreting the results of studies that undertook reconstruction using rhBMP-2, rhBMP-7, and 'stem cell based' treatment, a pertinent point is that multiple author's implemented supra-physiological doses of these proteins in order to achieve acceptable bone healing in a bovine collagen type-1 carrier [31, 33•, 34, 42-44]. Although collagen sponges have been shown to increase retention of osteogenic growth factors by $15-55 \%$ [55], safety concerns regarding use of milligram doses of rhBMP have been raised, eliciting effects such as oedema, ectopic bone formation, erythema and local inflammation $[56,57]$. Despite the undoubted osteogenic potential of
BMSCs, further large animal studies are required to elucidate their safety profile [35].

Only one study showed a treatment that was able to replicate the mechanical properties of the un-operated contralateral mandible [38•]. This may be have been due to the differing sacrifice times used across the studies; and thus, more effort should be made to standardize this for the sake of direct comparison between studies. No other study employing tissue engineering was able to show results comparable to a nonoperated mandible or autologous graft positive control. Studies of transport-disc-distraction osteogenesis treatment of continuity defects reported a discontinuity defect in at least one of the treatment group subjects, as well as additional complications [43, 58]. Furthermore, studies used small sample sizes $(n=6)$ bar one exception $(n=12)$ [38•].

Despite the growing number of animal studies investigating the restoration of critical sized mandibular defects, there remains a high variability between factors influencing studies. These factors include the animal model, defect site, defect size, sacrifice time, and postexplantation analysis. Furthermore, only three of the studies incorporated a 'gold standard' autologous graft as a positive control [36, 39, 41]. The heterogeneity of the studies makes comparison between different treatment modalities difficult. It is therefore impossible to assess if one treatment is superior to another by direct comparison. Ideally, the future assessment of new treatment modalities regarding bridging of critical sized mandibular defects should be carried out in a standardized large animal model employing a continuity defect. This model should feature an adequate autologous graft as a positive control. Post-explantation analysis should be comprehensive and include $\mu \mathrm{CT}$, radiographic, histological and histomorphometric analysis, as well as biomechanical testing.

Due to the inherent heterogeneity between studies, it is problematic for clinicians and researchers alike to compare the many possible combinations of scaffold/ carrier and autologous bone precursor cells or osteogenic growth factors. Despite tissue engineered constructs and transport-disc-distraction osteogenesis demonstrating some potential regarding their use for bridging critical sized mandibular defects, when compared to the current standard of care, there is currently not enough evidence within the literature for these treatments to be used ahead of vascularized bone grafts. TDDO and TE treatments have not yet undergone enough rigorous testing to warrant translation into the clinic and therefore do not currently have the capabilities to replace the current 'gold standard' of care.

Acknowledgments The authors would like to thank Professor Dietmar Hutmacher for his help and guidance. 


\section{Compliance with Ethical Standards}

Conflict of Interest Gary.I Brierly, Seamus Tredinnick, Anthony.J Lynham and Maria.A Woodruff declare that they have no conflicts of interest.

Human and Animal Rights and Informed Consent This article does not contain any studies with humans or animal subjects performed by any of the authors.

\section{References}

Papers of particular interest, published recently, have been highlighted as:

- Of importance

1. Schmitz JP, Hollinger JO. The critical size defect as an experimental model for craniomandibulofacial nonunions. Clin Orthop Relat Res. 1986;299-308.

2. Hollinger JO, Kleinschmidt JC. The critical size defect as an experimental model to test bone repair materials. J Craniofac Surg. 1990;1:60-8.

3. Bell RB, Gregoire C. Reconstruction of mandibular continuity defects using recombinant human bone morphogenetic protein 2: a note of caution in an atmosphere of exuberance. J Oral Maxillofac Surg. 2009;67:2673-8. This article discusses the risks and benefits of using rhBMP-2 for mandibular reconstruction.

4. Bak M, Jacobson AS, Buchbinder D, Urken ML. Contemporary reconstruction of the mandible. Oral Oncol. 2010;46:71-6.

5. Goh BT, Lee S, Tideman H, Stoelinga PJ. Mandibular reconstruction in adults: a review. Int J Oral Maxillofac Surg. 2008;37:597605.

6. Sacco AG, Chepeha DB. Current status of transport-disc-distraction osteogenesis for mandibular reconstruction. Lancet Oncol. 2007;8: 323-30.

7. Langer R, Vacanti JP. Tissue engineering. Science. 1993;260: $920-6$

8. Sokolsky-Papkov M, Agashi K, Olaye A, Shakesheff K, Domb AJ. Polymer carriers for drug delivery in tissue engineering. Adv Drug Deliv Rev. 2007;59:187-206.

9. Weinand C, Pomerantseva I, Neville CM, Gupta R, Weinberg E, Madisch I, et al. Hydrogel-beta-TCP scaffolds and stem cells for tissue engineering bone. Bone. 2006;38:555-63.

10. Schmidmaier G, Capanna R, Wildemann B, Beque T, Lowenberg D. Bone morphogenetic proteins in critical-size bone defects: what are the options? Injury. 2009;40 Suppl 3:S39-43.

11. Alvarez P, Hee CK, Solchaga L, Snel L, Kestler HK, Lynch SE, et al. Growth factors and craniofacial surgery. J Craniofac Surg. 2012;23:20-9.

12. Hutmacher DW. Scaffolds in tissue engineering bone and cartilage. Biomaterials. 2000;21:2529-43.

13. Reichert JC, Saifzadeh S, Wullschleger ME, Epari DR, Schutz MA, Duda GN, et al. The challenge of establishing preclinical models for segmental bone defect research. Biomaterials. 2009;30:2149-63.

14. Costantino PD, Friedman CD, Shindo ML, Houston G, Sisson Sr GA. Experimental mandibular regrowth by distraction osteogenesis. Long-term results. Arch Otolaryngol Head Neck Surg. 1993;119:511-6.

15. Costantino PD, Shybut G, Friedman CD, Pelzer HJ, Masini M, Shindo ML, et al. Segmental mandibular regeneration by distraction osteogenesis. An experimental study. Arch Otolaryngol Head Neck Surg. 1990;116:535-45.

16. Elsalanty ME, Malavia V, Zakhary I, Mulone T, Kontogiorgos ED, Dechow PC, et al. Dentate transport discs can be used to reconstruct large segmental mandibular defects. J Oral Maxillofac Surg. 2015;73:745-58.

17. Ayoub AF, Richardson W, Koppel D, Thompson H, Lucas M, Schwarz T, et al. Segmental mandibular reconstruction by microincremental automatic distraction osteogenesis: an animal study. Br J Oral Maxillofac Surg. 2001;39:356-64.

18. Wang C, Zeng RS, Wang JN, Huang HZ, Sun J, Luo ZB. The study of distraction osteogenesis with nitinol shape memory alloy spring controlled by infrared light. Oral Surg Oral Med Oral Pathol Oral Radiol Endod. 2010;110:157-66.

19. Shang H, Lin X, Du J, He L, Liu Y. Use of a new curvilinear distractor to repair mandibular defects in dogs. Br J Oral Maxillofac Surg. 2012;50:166-70.

20. Elsalanty ME, Zakhary I, Akeel S, Benson B, Mulone T, Triplett GR, et al. Reconstruction of canine mandibular bone defects using a bone transport reconstruction plate. Ann Plast Surg. 2009;63:441-8.

21. Nagashima LK, Rondon-Newby M, Zakhary IE, Nagy WW, Zapata $\mathrm{U}$, Dechow PC, et al. Bone regeneration and docking site healing after bone transport distraction osteogenesis in the canine mandible. J Oral Maxillofac Surg. 2012;70:429-39.

22. Hu YC, Liu X, Shen JJ, He JC, Chen QE. Experimental study of canine bone marrow mesenchymal stem cells combined with calcium phosphate cement for repair of mandibular bone defects in Beagle dogs. Shanghai Kou Qiang Yi Xue. 2014;23:402-8.

23. Xu M, Zhang X, Meng S, Dai X, Han B, Deng X. Enhanced critical size defect repair in rabbit mandible by electrospun gelatin/ $\beta$-TCP composite nanofibrous membranes. J Nanomater. 2015.

24. Parrilla C, Saulnier N, Bernardini C, Patti R, Tartaglione T, Fetoni $\mathrm{AR}$, et al. Undifferentiated human adipose tissue-derived stromal cells induce mandibular bone healing in rats. Arch Otolaryngol Head Neck Surg. 2011;137:463-70.

25. Liao HT, Chen CT, Chen CH, Chen JP, Tsai JC. Combination of guided osteogenesis with autologous platelet-rich fibrin glue and mesenchymal stem cell for mandibular reconstruction. J Trauma. 2011;70:228-37.

26. Streckbein P, Jackel S, Malik CY, Obert M, Kahling C, Wilbrand JF, et al. Reconstruction of critical-size mandibular defects in immunoincompetent rats with human adipose-derived stromal cells. J Craniomaxillofac Surg. 2013;41:496-503. This study demonstrates the regeneration of non continuity critical sized defects.

27. Guo J, Meng Z, Chen G, Xie D, Chen Y, Wang H, et al. Restoration of critical-size defects in the rabbit mandible using porous nanohydroxyapatite-polyamide scaffolds. Tissue Eng Part A. 2012;18:1239-52.

28. Busuttil Naudi K, Ayoub A, McMahon J, Di Silvio L, Lappin $\mathrm{D}$, Hunter KD, et al. Mandibular reconstruction in the rabbit using beta-tricalcium phosphate (beta-TCP) scaffolding and recombinant bone morphogenetic protein 7 (rhBMP-7) - histological, radiographic and mechanical evaluations. J Craniomaxillofac Surg. 2012;40:e461-9. This study is a recent use of rhBMP-7 for the rgeneration of mandibular segmental defects.

29. Alfotawei R, Naudi KB, Lappin D, Barbenel J, Di Silvio L, Hunter $\mathrm{K}$, et al. The use of TriCalcium Phosphate (TCP) and stem cells for the regeneration of osteoperiosteal critical-size mandibular bony defects, an in vitro and preclinical study. J Cranio-Maxillofac Surg. 2014;42:863-9. This study shows the recent use of stem cells for attempted mandibular regeneration.

30. Davies SD, Ochs MW. Bone morphogenetic proteins in craniomaxillofacial surgery. Oral Maxillofac Surg Clin North Am. 2010;22:17-31. 
31. Hussein KA, Zakhary IE, Elawady AR, Emam HA, Sharawy M, Baban B, et al. Difference in soft tissue response between immediate and delayed delivery suggests a new mechanism for recombinant human bone morphogenetic protein 2 action in large segmental bone defects. Tissue Eng Part A. 2012;18:665-75.

32. Hussein KA, Zakhary IE, Hailat D, Elrefai R, Sharawy M, Elsalanty ME. Delayed versus immediate reconstruction of mandibular segmental defects using recombinant human bone morphogenetic protein 2/absorbable collagen sponge. J Oral Maxillofac Surg. 2013;71:1107-18.

33. Herford AS, Lu M, Buxton AN, Kim J, Henkin J, Boyne PJ, et al. Recombinant human bone morphogenetic protein 2 combined with an osteoconductive bulking agent for mandibular continuity defects in nonhuman primates. J Oral Maxillofac Surg. 2012;70:703-16. This study shows the recent use of rhBMP-2 for reconstruction of mandibular continuity defects.

34. Marukawa E, Asahina I, Oda M, Seto I, Alam M, Enomoto S. Functional reconstruction of the non-human primate mandible using recombinant human bone morphogenetic protein-2. Int $\mathrm{J}$ Oral Maxillofac Surg. 2002;31:287-95.

35. Kimelman N, Pelled G, Helm GA, Huard J, Schwarz EM, Gazit D. Review: gene- and stem cell-based therapeutics for bone regeneration and repair. Tissue Eng. 2007;13:1135-50.

36. Wu W, Chen X, Mao T, Chen F, Feng X. Bone marrow-derived osteoblasts seeded into porous beta-tricalcium phosphate to repair segmental defect in canine's mandibula. Ulus Travma Acil Cerrahi Derg. 2006;12:268-76.

37. He Y, Zhang ZY, Zhu HG, Qiu W, Jiang X, Guo W. Experimental study on reconstruction of segmental mandible defects using tissue engineered bone combined bone marrow stromal cells with three-dimensional tricalcium phosphate. J Craniofac Surg. 2007;18:800-5.

38. Yuan J, Zhang WJ, Liu G, Wei M, Qi ZL, Liu W, et al. Repair of canine mandibular bone defects with bone marrow stromal cells and coral. Tissue Eng Part A. 2010;16:1385-94. Important article demonsrrating the ideal post explantation analysis of animal models.

39. Yuan J, Cui L, Zhang WJ, Liu W, Cao Y. Repair of canine mandibular bone defects with bone marrow stromal cells and porous betatricalcium phosphate. Biomaterials. 2007;28:1005-13.

40. Schliephake H, Knebel JW, Aufderheide M, Tauscher M. Use of cultivated osteoprogenitor cells to increase bone formation in segmental mandibular defects: an experimental pilot study in sheep. Int J Oral Maxillofac Surg. 2001;30:531-7.

41. Strong EB, Rubinstein B, Pahlavan N, Martin B, KuntsiVaattovaara $\mathrm{H}$, Verstraete FJ. Mandibular reconstruction with an alloplastic bone tray in dogs. Otolaryngol Head Neck Surg. 2003;129:417-26.

42. Forriol F, Longo UG, Concejo C, Ripalda P, Maffulli N, Denaro V. Platelet-rich plasma, rhOP-1 (rhBMP-7) and frozen rib allograft for the reconstruction of bony mandibular defects in sheep. A pilot experimental study. Injury. 2009;40(3):S44-9.

43. Ayoub A, Challa SR, Abu-Serriah M, McMahon J, Moos K, Creanor $\mathrm{S}$, et al. Use of a composite pedicled muscle flap and rhBMP-7 for mandibular reconstruction. Int J Oral Maxillofac Surg. 2007;36: 1183-92.

44. Abu-Serriah M, Kontaxis A, Ayoub A, Harrison J, Odell E, Barbenel J. Mechanical evaluation of mandibular defects reconstructed using osteogenic protein-1 (rhOP-1) in a sheep model: a critical analysis. Int J Oral Maxillofac Surg. 2005;34:287-93.

45. Ilizarov GA. The tension-stress effect on the genesis and growth of tissues. Part I. The influence of stability of fixation and soft-tissue preservation. Clin Orthop Relat Res. 1989;249-81.

46. Schrag C, Chang YM, Tsai CY, Wei FC. Complete rehabilitation of the mandible following segmental resection. J Surg Oncol. 2006;94:538-45.

47. Hayden RE, Mullin DP, Patel AK. Reconstruction of the segmental mandibular defect: current state of the art. Curr Opin Otolaryngol Head Neck Surg. 2012;20:231-6. This paper presents a recent review of mandibular reconstruction techniques.

48. Muschler GF, Raut VP, Patterson TE, Wenke JC, Hollinger JO. The design and use of animal models for translational research in bone tissue engineering and regenerative medicine. Tissue Eng Part B Rev. 2010;16:123-45.

49. Zapata U, Halvachs EK, Dechow PC, Elsalanty ME, Opperman LA. Architecture and microstructure of cortical bone in reconstructed canine mandibles after bone transport distraction osteogenesis. Calcif Tissue Int. 2011;89:379-88.

50. Kontogiorgos E, Elsalanty ME, Zapata U, Zakhary I, Nagy WW, Dechow PC, et al. Three-dimensional evaluation of mandibular bone regenerated by bone transport distraction osteogenesis. Calcif Tissue Int. 2011;89:43-52.

51. Giotakis N, Narayan B, Nayagam S. Distraction osteogenesis and nonunion of the docking site: is there an ideal treatment option? Injury. 2007;38:S100-S7.

52. Zapata U, Elsalanty ME, Dechow PC, Opperman LA. Biomechanical configurations of mandibular transport distraction osteogenesis devices. Tissue Eng Part B Rev. 2010;16:273-83.

53. Zhang $Z$. Bone regeneration by stem cell and tissue engineering in oral and maxillofacial region. Front Med. 2011;5:401-13.

54. Cancedda R, Giannoni P, Mastrogiacomo M. A tissue engineering approach to bone repair in large animal models and in clinical practice. Biomaterials. 2007;28:4240-50.

55. Hollinger JO, Schmitt JM, Buck DC, Shannon R, Joh SP, Zegzula $\mathrm{HD}$, et al. Recombinant human bone morphogenetic protein- 2 and collagen for bone regeneration. J Biomed Mater Res. 1998;43:356 64.

56. Luginbuehl V, Meinel L, Merkle HP, Gander B. Localized delivery of growth factors for bone repair. Eur J Pharm Biopharm. 2004;58: 197-208.

57. Haidar ZS, Hamdy RC, Tabrizian M. Delivery of recombinant bone morphogenetic proteins for bone regeneration and repair. Part A: Current challenges in BMP delivery. Biotechnol Lett. 2009;31: 1817-24.

58. Fennis JP, Stoelinga PJ, Merkx MA, Jansen JA. Reconstruction of the mandible with a poly(D, L-lactide) scaffold, autogenous corticocancellous bone graft, and autogenous platelet-rich plasma: an animal experiment. Tissue Eng. 2005;11:1045-53. 